\title{
Electroretinography and Visual Evoked Potentials in Childhood Brain Tumor Survivors
}

\section{Pietila, Sari}

2016-07

Pietila , S , Lenko , H L , Oja , S , Koivisto , A-M , Pietila , T \& Mäkipernaa , A M-T 2016 , ' Electroretinography and Visual Evoked Potentials in Childhood Brain Tumor Survivors ', Journal of Child Neurology, vol. 31 , no. 8 , pp. 998-1004 . https://doi.org/10.1177/0883073816634863

http://hdl.handle.net/10138/224074

https://doi.org/10.1177/0883073816634863

publishedVersion

Downloaded from Helda, University of Helsinki institutional repository.

This is an electronic reprint of the original article.

This reprint may differ from the original in pagination and typographic detail.

Please cite the original version. 


\title{
Electroretinography and Visual Evoked Potentials in Childhood Brain Tumor Survivors
}

\author{
Sari Pietilä, MD, PhD', Hanna L. Lenko, MD, $\mathrm{PhD}^{2}$, \\ Sakari Oja, MD, $\mathrm{PhD}^{2}$, Anna-Maija Koivisto, $\mathrm{MSc}^{3}$, \\ Timo Pietilä, $\mathbf{M D}^{4}$, and Anne Mäkipernaa, MD, $\mathrm{PhD}^{5}$
}

\begin{abstract}
This population-based cross-sectional study evaluates the clinical value of electroretinography and visual evoked potentials in childhood brain tumor survivors. A flash electroretinography and a checkerboard reversal pattern visual evoked potential (or alternatively a flash visual evoked potential) were done for $5 \mathrm{I}$ survivors (age 3.8-28.7 years) after a mean follow-up time of 7.6 (I.5-I5.I) years. Abnormal electroretinography was obtained in I case, bilaterally delayed abnormal visual evoked potentials in $22 /$ $5 \mathrm{I}(43 \%)$ cases. Nine of 25 patients with infratentorial tumor location, and altogether 12 out of 31 (39\%) patients who did not have tumors involving the visual pathways, had abnormal visual evoked potentials. Abnormal electroretinographies are rarely observed, but abnormal visual evoked potentials are common even without evident anatomic lesions in the visual pathway. Bilateral changes suggest a general and possibly multifactorial toxic/adverse effect on the visual pathway. Electroretinography and visual evoked potential may have clinical and scientific value while evaluating long-term effects of childhood brain tumors and tumor treatment.
\end{abstract}

\section{Keywords}

brain tumor, childhood, late effects, visual evoked potentials, electroretinography

Received November 29, 2015. Received revised January 9, 2016. Accepted for publication January I8, 2016.

Brain tumors are the most common solid tumors in children, and the second most common childhood cancer. ${ }^{1}$ Survival rates among childhood brain tumor patients have improved over recent decades; this is related to advances in diagnosis and treatment. ${ }^{2}$ Survivors are at a high risk of chronic health conditions later in life. ${ }^{3}$ Treatment modalities include surgery, radiotherapy and chemotherapy. Adverse late effects may be due to the tumor itself or consequences of the treatment. Cranial nerve dysfunctions and visual problems are common in childhood brain tumor survivors. ${ }^{4,5}$

Tumor compression, tumor growth/invasion or surgical intervention can damage the optic nerve or other parts of the visual pathway. Ophthalmic complications have been found in patients with hydrocephalus, raised intracranial pressure, and shunt malfunction, and also in patients with slit-ventricle syndrome. ${ }^{6,7}$ Radiotherapy of the head and of the neck may cause optic neuropathy. ${ }^{8}$ Optic neuropathy can also be due to toxins. ${ }^{9}$ Radiation damage could be acute, or symptoms may develop from months to years following treatment. ${ }^{8,10}$ A radiation retinopathy is a possible complication of radiation therapy. ${ }^{11,12}$ The posterior retina is more sensitive to radiation than the peripheral retina. ${ }^{11}$ Factors that have been shown to increase the likelihood of radiation retinopathy include chemotherapy and vascular diseases, such as diabetes and hypertension. ${ }^{11} \mathrm{~A}$ common feature of different optic neuropathies is retinal ganglion cell apoptosis and axonal damage. ${ }^{13}$ In addition to the functioning of rods and cones, an electroretinography gives information of the functioning of other retinal cells, including retinal ganglion cells. ${ }^{13,14}$ On the other hand, visual evoked potentials are useful to quantify nerve dysfunction from the retina to the visual cortex, and retinal disease alone may alter visual evoked potentials. ${ }^{15}$ Visual evoked potential tests have been used for the evaluation of tumor progression in optic pathway gliomas. ${ }^{16,17}$

\footnotetext{
'Rinnekoti Foundation, Espoo, Finland

${ }^{2}$ Department of Pediatrics, Tampere University Hospital, Tampere, Finland

${ }^{3}$ School of Health Sciences, University of Tampere, Tampere, Finland

${ }^{4}$ Department of Neurology, Hatanpää Hospital, Tampere, Finland

${ }^{5}$ Department of Hematology, Cancer Center, Helsinki University Hospital, Helsinki University, Helsinki, Finland
}

\section{Corresponding Author:}

Sari Pietilä, MD, PhD, Rinnekoti Foundation, Rinnekodintie 10, 02980 Espoo, Finland.

Email: sari.pietila@sci.fi 
The purpose of this study was to examine the prevalence of abnormal functioning of the retina with an electroretinography, and to examine long-term damage of the visual pathway with a visual evoked potential test in childhood brain tumor survivors. Also, the purpose was to identify factors associated with problems discovered.

\section{Material and Methods}

\section{Subjects}

A total of 104 primary brain tumor patients diagnosed at younger than 17 years of age between the years 1983 and 1997 were treated in Tampere University Hospital. Of the 80 survivors, 75 potentially eligible patients were invited to participate in this population-based cross-sectional study. Five patients were excluded as the study protocol was considered too demanding for them. Fifty-two (69\%) participated in the study and were examined at a mean age of 14.2 years (range 3.8-28.7 years) after a mean follow-up time of 7.5 years (1.515.1 years) (Table 1$)$. In case of refusal to participate, the reason was not asked.

\section{Treatment of the Tumor and Hydrocephalus}

Diagnosed brain tumors included both benign and malignant tumors. The most common single tumor type was pilocytic astrocytoma. Tumor localization is presented in Table 1. All 52 participants had undergone 1 or more surgical procedures (Table 1). Twenty-nine $(56 \%)$ were treated by surgery only. In addition to surgical treatment, radiotherapy and/or chemotherapy were given (Table 1). Thirteen (25\%) participants had a relapse or residual tumor upon evaluation, while only 3 of them had a tumor location in the visual pathway. Thirty $(58 \%)$ participants had been diagnosed with hydrocephalus; all except 1 had been shunted, and $59 \%$ of them had needed shunt revisions (Table 1). Shunt dysfunction was the primary reason for revision; only 3 patients experienced shunt infections.

Of the $17(33 \%)$ children who received chemotherapy, 10 were on an " 8 drugs in 1 day" protocol, and 8 children received chemotherapy according to other protocols. One of these 8 patients also received an " 8 in 1 " chemotherapy, and 1 chemotherapy according to 2 other different protocols. In the " 8 in 1 " protocol, 7 cytostatic drugs (lomustine [CCNU], vincristine, hydroxyurea, procarbazine, cisplatin, cytarabine [ARA-C], and either dacarbazine or cyclophospamide) and methylprednisolone were administered. ${ }^{18,19}$ Other combinations were bleomycin and CCNU (one patient), vincristine and CCNU plus prednisolone or dexametasone (2 patients), bleomycin and etoposide (VP-16) plus cisplatin (one patient), bleomycin, vinblastine, and teniposide (VM-26) plus cisplatin (one patient), vincristine, VP-16, and cyclophosphamide plus cisplatin (2 patients) followed by maintenance with vincristine, VP-16, and karboplatin (and cyclophosphamide). Due to progressive disease, 2 patients received oral VP-16 treatment. Glucocorticoids in variable doses and courses were included in the treatment of all patients, even those with only operation.

\section{Treatment of Epilepsy}

Seven patients $(13 \%)$ received antiepileptic medication during the study. Two patients had carbamazepine, 1 valproate, 1 carbamazepine and valproate, 1 lamotrigine and topiramate, 1 oxcarbazine, and 1 vigabatrin and oxcarbazine medications.
Table I. Clinical Data on the 52 Brain Tumor Patients Diagnosed Below 17 Years of Age.

\begin{tabular}{|c|c|}
\hline Median age at diagnosis, years & $6.0(0.1-15.5)$ \\
\hline Mean follow-up time, years & $7.5(1.5-15.1)$ \\
\hline Mean time after treatment, years & $6.2(1.2-14.8)$ \\
\hline Mean age at evaluation, years & | $4.2(3.8-28.7)$ \\
\hline Sex, male/female & $27 / 25$ \\
\hline Neurofibromatosis & $3(6)$ \\
\hline \multicolumn{2}{|l|}{ Site of the tumor } \\
\hline Infratentorial & $25(48)$ \\
\hline Brain stem & $5(10)$ \\
\hline Supratentorial & $27(52)$ \\
\hline Cerebral hemisphere & $13(25)$ \\
\hline Occipital & $\mathrm{I}(2)$ \\
\hline Central location & $14(27)$ \\
\hline Pituitary & I (2) \\
\hline Hypothalamus & $5(10)$ \\
\hline Chiasma opticum & I (2) \\
\hline Corpus pineale & $2(4)$ \\
\hline Resection/biopsy & $5 \mathrm{I} / \mathrm{I}(98 / 2)$ \\
\hline Grossly total & $30(58)$ \\
\hline Partial & $21(40)$ \\
\hline Reoperation/biopsy & $17 / 1(33 / 2)$ \\
\hline I & $\mathrm{I} I / \mathrm{I}(2 \mathrm{I} / 2)$ \\
\hline 2 & $6(12)$ \\
\hline Severe perioperative complications ${ }^{a}$ & $15(29)$ \\
\hline Chemotherapy & $17(33)$ \\
\hline Radiotherapy & $20(38)$ \\
\hline Median age at start of radiotherapy, years & $7.2(0.2-20.9)$ \\
\hline Cranial & $12(23)$ \\
\hline Local & $9(17)$ \\
\hline Cranial only & $3(6)$ \\
\hline Including hypothalamo-pituitary axis & II (2I) \\
\hline Craniospinal & $8(15)$ \\
\hline Including hypothalamo-pituitary axis & $8(15)$ \\
\hline Median tumor dose, Gy & $50.5(16.0-60.0)$ \\
\hline Both chemotherapy and radiotherapy & 14 (27) \\
\hline Recidive or residual tumor at evaluation & $13(25)$ \\
\hline Hydrocephalus & $30(58)$ \\
\hline Shunted hydrocephalus & $29(56)$ \\
\hline Shunt revisions & $17(33)$ \\
\hline I & $7(14)$ \\
\hline 2 & $6(12)$ \\
\hline 3 & $2(4)$ \\
\hline More than 5 & $2(4)$ \\
\hline Some kind of shunt at evaluation & $27(52)$ \\
\hline
\end{tabular}

Categorical data are given as number of patients (\%), continuous data as mean or median (range).

${ }^{a}$ Need of multiple operations, shunt revisions, meningitis, ventriculitis with ventriculostomy, expanding intracranial effusions or cysts, prolonged impaired consciousness, intractable seizures and neurological deficits.

\section{Procedure and Materials}

Patients' medical records were checked for clinical history, and if necessary, their medical records were completed by data from other hospitals. The patients were examined by the same physician (SP). The neurological examination included visual acuity testing, visual fields with confrontational visual field exam, estimation of strabismus with cover test, eye movements and diplopia, eyelid motorics, pupillary reflexes, and fundus examination by ophthalmoscope. The E chart was used for visual acuity testing. One eye was covered at a time and 
the vision of each eye was recorded separately, followed by testing of vision of both eyes together. Simultaneous visual acuity of both eyes $<$ 0.5 was defined as impaired. Information of the use of eyeglasses was collected.

The flash electroretinography and the checkerboard reversal pattern visual evoked potential (or alternatively the flash visual evoked potential) were done in the Department of Clinical Neurophysiology of Tampere University Hospital using the same recording setups as in routine clinical diagnostic measurements. The flash electroretinography protocol was a 5-stage measurement where the responses of the rod and the cone receptors of the retina could be assessed separately. ${ }^{20}$ The most specific response of rod receptors was obtained using a single dim flash stimulus after dark adaptation, and the most cone specific response was obtained using a flicker of $30 \mathrm{~Hz}$ after light adaptation. Corneal electrodes (referenced to the lateral corner of the ipsilateral eye) were primarily used for electroretinography testing. The pupils were dilated during the recording of flash electroretinographies. A Nicolet Ganzfeld stimulator (Natus Medical Incorporated, Pleasanton, CA, USA) was used to generate the flash stimuli in electroretinography recordings and flash visual evoked potential recordings. Recordings were analyzed with an 8-channel Nicolet Viking II ENMG/EP system (Natus Medical Incorporated, Pleasanton, CA, USA), which was also used in all visual evoked potential recordings. Pattern visual evoked potential was the preferred method used to asses the optic pathway, but flash visual evoked potential was used as a secondary choice if the pattern visual evoked potential was not measurable. This could result from poor cooperation, poor vision, or often difficulties in focusing and keeping the line of sight of the eye steady in the centre of the 30 inch Finnvideo monitor driven by Nicolet NIC 1015 visual stimulator (Natus Medical Incorporated, Pleasanton, CA, USA). The check pattern was 32 blocks/line ( 8 min of visual arc). The measurement distance between the monitor and the patient was 1.5 meters. The electrode setup in the visual evoked potential measurements was a commonly used 3-channel recording (active $\mathrm{Oz}, \mathrm{Oz}+3$ $\mathrm{cm}$ left, $\mathrm{Oz}+3 \mathrm{~cm}$ right, reference $\mathrm{Fz}$ ). The upper normal limits for the checkerboard reversal pattern visual evoked potential was $85 \mathrm{~ms}$ for the N75 latency, $113 \mathrm{~ms}$ for the P100 latency and $165 \mathrm{~ms}$ for the N145 latency. There is a rough correspondence between N75, P100, and N145 latencies in the pattern visual evoked potential and N1, P2, and N3 latencies in the flash visual evoked potential. However, since the retinal structures and components of the optic pathways that carry the signal are not the same when using flash or pattern stimulus, the normal limits for the flash visual evoked potential are not similar to the pattern visual evoked potential. The upper normal limits for the flash visual evoked potential was $80 \mathrm{~ms}$ for the $\mathrm{N} 1$ latency and $150 \mathrm{~ms}$ for the P2 latency. The P100 (or P2) latency was the most important component in the visual evoked potential analysis. The recordings were analyzed by Sakari Oja, MD, PhD.

If electroretinography and/or visual evoked potential were abnormal, the patient was referred to the ophthalmologist for an examination of visual fields and of color vision. Also, if there was a suspicion of a new visual field defect in the clinical examination, or if other problems were discovered, an ophthalmologist was consulted. Patients' existing ophthalmological medical records were also used in the estimation of the visual field defect.

\section{Statistical Analysis}

Continuous data were analyzed using the independent sample t-test in case of normal distribution, and the Mann-Whitney $U$ test or the Kruskal-Wallis test in case of skewed distribution. Categorized data were analyzed using Fisher's exact test or the $\chi^{2}$ test. The Spearman correlation coefficient was used for correlation estimates because of the skewed distribution of the analyzed data. A $P$ value less than .05 was considered significant. Statistical analyses were carried out using the SPSS for Windows versions 10.0, 16.0, 17.0, 18.0, and 20.0.

\section{Results}

The electroretinography and visual evoked potential tests were done for 51 childhood brain tumor survivors 1.5-15.1 years (mean 7.6 years) after the diagnosis. In 1 case, the cooperation was insufficient to perform the tests.

Electroretinography was slightly abnormal (bilaterally delayed b-wave latencies, but normal amplitudes) only in $2 \%$ (1/51) of the cases. The patient with abnormal electroretinography had a tumor involving the visual pathway, had not received radiotherapy or chemotherapy and did not have antiepileptic medication, but had neurofibromatosis and hydrocephalus and had gone through several shunt revisions. The patient's flash visual evoked potential was also abnormal (pattern visual evoked potential was unmeasurable). The patient's vision was $0.1 / 0.1$ (both eyes 0.1 ). In repeated ophthalmologic examinations the patient's visual fields had a concentric defect, but were within normal limits and the ocular fundus were normal. The color vision was not examined.

Abnormal, bilaterally delayed visual evoked potentials were obtained in 22/51 (43\%) cases. In 41 cases the visual evoked potential results were based on pattern visual evoked potential, and in 9 cases on flash visual evoked potential. In 1 case, the results were unreliable even though both the pattern and the flash visual evoked potential were done (Table 2). Between the patients' right and left eyes the correlation for the N75(N1) latency was $.847(\mathrm{n}=40)$, the correlation for the P100(P2) latency was $.920(\mathrm{n}=48)$ and for the N145(N3) latency .736 $(\mathrm{n}=43)$. One patient was blind. His flash visual evoked potential showed a partly functional optical nerve on the right side, but no cortical activity on the left side.

The age at diagnosis $(P=.358)$ and the follow-up time $(P=$ .400) were not statistically significantly associated with abnormal visual evoked potentials. Ten out of $20(50 \%)$ patients who had tumors involving the visual pathways had abnormal visual evoked potentials (Table 2). Visual evoked potentials were abnormal in all patients with chiasmatic, hypophyseal, or pineal tumor location, in most patients with hypothalamic tumor location, and in singular patients with tumor located in third ventricle, temporal or parietal lobe, but were normal in some patients with hypothalamic tumor location and in a few patients who had tumors in third ventricle, occipital or temporal lobe. Altogether 12/31 (39\%) of the patients who did not have tumors involving the visual pathways had also abnormal visual evoked potential responses (Table 2). It is notable, that 9 out of $25(36 \%)$ patients with infratentorial tumor location had abnormal visual evoked potentials. The occurrence of residual/recidive tumor during the study was not statistically significantly associated with abnormal visual evoked potentials $(P=.520)$. 
Table 2. Visual Evoked Potential (VEP) Test Results of the 5 I Childhood Brain Tumor Survivors.

\begin{tabular}{|c|c|c|c|c|c|c|c|c|c|}
\hline Explanatory variable & \multicolumn{9}{|c|}{ Outcome variable } \\
\hline r location & & & $P$ & & & $P$ & & & $P$ \\
\hline Supratentorial $(\mathrm{n}=26)$ & 112 & $(97-176)(n=20)$ & .686 & 109 & $(99-173)(n=20)$ & .814 & 13 & $(50)$ & .407 \\
\hline Infratentorial $(n=25)$ & 108 & $(96-156)(n=21)$ & & 108 & $(99-130)(n=21)$ & & 9 & $(38)^{c}$ & \\
\hline \multicolumn{10}{|l|}{ Tumor location } \\
\hline \multicolumn{10}{|l|}{ Chemotherapy } \\
\hline Yes $(n=16)$ & 107 & $(100-156)(n=13)$ & .750 & 110 & $(99-134)(n=13)$ & .570 & 6 & $(40)^{c}$ & .765 \\
\hline No $(n=35)$ & III & $(96-176)(n=28)$ & & 107 & $(99-173)(n=28)$ & & 16 & $(46)$ & \\
\hline \multicolumn{10}{|l|}{ Cranial irradiation } \\
\hline Yes $(n=19)^{d}$ & 115 & $(100-176)(n=17)$ & .277 & 110 & $(99-173)(n=17)$ & .213 & 9 & $(50)^{c}$ & .565 \\
\hline No $(n=32)$ & 110 & $(96-126)(n=24)$ & & 106 & $(99-129)(n=24)$ & & 13 & $(4 I)$ & \\
\hline \multicolumn{10}{|l|}{ Hydrocephalus } \\
\hline Yes $(n=29)$ & 107 & $(96-156)(n=22)$ & .313 & 107 & $(99-134)(n=22)$ & .402 & II & $(39)^{c}$ & .568 \\
\hline No $(n=22)$ & 112 & $(97-176)(n=19)$ & & 113 & $(99-173)(n=19)$ & & II & (50) & \\
\hline \multicolumn{10}{|l|}{ Shunt revisions } \\
\hline Yes $(n=16)$ & 112 & $(100-156)(n=11)$ & .674 & 108 & $(100-130)(n=11)$ & .942 & 7 & $(44)$ & 1.000 \\
\hline No $(n=35)$ & 109 & $(96-176)(n=30)$ & & 109 & $(99-173)(n=30)$ & & 15 & $(44)^{c}$ & \\
\hline \multicolumn{10}{|l|}{ Antiepileptic medication } \\
\hline Yes $(n=6)$ & 112 & $(104-124)(n=5)$ & .743 & 113 & $(101-133)(n=5)$ & .473 & 3 & $(50)$ & 1.000 \\
\hline No $(n=45)$ & 110 & $(96-176)(n=36)$ & & 108 & $(99-173)(n=5)$ & & 19 & $(43)^{c}$ & \\
\hline
\end{tabular}

Categorical data are given as number of patients (\%), continuous data as median (range).

${ }^{a}$ Not reported in 9 cases where results based on flash VEP and in I case with unreliable test results.

${ }^{b}$ Abnormal results of both pattern and flash VEP tests are included.

${ }^{\mathrm{C}}$ The test results were unreliable in I case and are not included in statistical analyses.

${ }^{\mathrm{d}}$ All analyzed patients received radiation to the hypothalamic-pituitary axis.

The patient who had vigabatrin medication had an abnormal visual evoked potential while the electroretinography was normal. Her tumor was attached to the optic nerve, but the nerve was released in the operation. She had received radiotherapy and chemotherapy, but did not have hydrocephalus. In the ophthalmologic examination there was a concentric visual field defect of the right eye. The visual field of the left eye could not be examined because of the oculomotor nerve paresis (ptosis, strabismus). The color vision was normal.

Of all 52 patients, $9(17 \%)$ had impaired vision $(<0.5)$, and 5 of them were among the patients with abnormal visual evoked potentials. Impaired vision was not statistically significantly associated with abnormal visual evoked potential responses $(P=.481)$. In addition to the tumor related causes of impaired vision, there were other reasons (like myopia) for a decreased visual acuity. Fifteen (29\%) patients had eyeglasses, but 6 of them did not use them.

Of all 52 patients, 46 (89\%) had received a postoperative ophthalmologic examination. In 6 cases there had been no ophthalmologic examination, or the information was not available. If the ophthalmologic examination of the visual fields was missing, the results of the confrontational visual field exam were used. Among the 22 patients with abnormal visual evoked potentials, 6 patients (27\%) had visual field defect, and in 1 case (the blind patient) it could not be examined. Among the 28 patients with normal visual evoked potentials, 2 patients $(7 \%)$ had a visual field defect (one had scotomas and the other had homonymous hemianopia). In 3 cases, the visual fields could not be examined or the results were uncertain. Even though visual field defects were more common in the group with abnormal visual evoked potentials than in the group with normal visual evoked potentials, no statistically significant association was found $(P=.117)$. The perimetry was performed usually by Goldmann method and in a few cases by Humphrey method. In some cases, also the ophthalmologists had used a confrontational visual field exam. Ptosis, strabismus, nystagmus, and difficulties in focusing and in concentration were reported as impairing factors in ophthalmologic examinations of visual fields.

Color vision was abnormal in 1 patient with an abnormal visual evoked potential test. Eleven patients with abnormal visual evoked potentials had a color vision that was considered to be normal, and the results of 9 patients were missing. In 1 case the color vision could not be examined.

Visual evoked potential tests had been done for 3 patients before this study, and an electroretinography for 1 patient. In 2 cases, the prior abnormal visual evoked potentials had 
normalized and in 1 case, with the tumor involving the visual pathway, the prior preoperative normal visual evoked potential test had turned to abnormal. This patient had impaired vision, optic atrophy, and visual field defect in the ophthalmological examination. Her color vision was in normal limits. The electroretinography of the 1 patient was normal both times.

\section{Discussion}

After a mean follow-up time of 7.6 years, visual evoked potentials were often abnormally delayed. This was the case in $43 \%$ of the childhood brain tumor survivors. The electroretinography was abnormal in only 1 case. It is reasonable to deduct that there is little direct effect of the tumor and its treatment to the retinal receptor level at least in this material.

In this study, visual evoked potentials were abnormal in all patients with chiasmatic, hypophyseal or pineal tumor location, and in most patients with hypothalamic tumor location. However, not every patient with a tumor involving particularly the latter parts of the optic pathway had abnormal visual evoked potentials. Also about $40 \%$ of the patients with other tumor locations, including patients with infratentorial tumor locations, had abnormal visual evoked potentials. Postchiasmatic parts of the optic tracts include signals originating from both eyes. Even when there is a clear lesion in the postchiasmatic part of the visual pathway in 1 side, the optic pathway in the other hemisphere might function quite normally. Since the visual evoked potential recordings (both pattern and flash visual evoked potential) represent signals that travel simultaneously through both sides of the postchiasmatic visual pathway, it is understandable that the prevalence of abnormal visual evoked potentials is not higher. Tumor size and tumor's invasivity might influence how serious the damage in the optic pathway is. Operative treatment may damage the optic pathway, but on the other hand operative treatment may release tumor compression of the optic pathway. Postoperative improvement was seen in visual fields and fundus examinations among some study patients with tumors involving the visual pathways.

Chemotherapeutics which have been reported to cause toxic optic neuropathy include vincristine, cisplatin, and carboplatin. $^{21-25}$ Caraceni et al $^{23}$ found that visual evoked potentials were first absent in a patient with optic neuropathy associated with cisplatin and carboplatin treatment. In following examinations, the visual evoked potentials were found to be present, but delayed. There have also been reports of retinal toxicity with electroretinography and visual evoked potential abnormalities after cisplatin treatment. ${ }^{26,27}$ It might be speculated that the presence of visual evoked potential abnormalities might hint to vulnerability for a certain drug. Antiepileptic vigabatrin induces retinopathy. ${ }^{28,29}$ Delayed visual evoked potentials are associated with elevations in intracranial pressure. ${ }^{15,30}$

In this study, cranial irradiation, chemotherapy, hydrocephalus, shunt revisions, and antiepileptic medication were not statistically significantly associated with abnormal visual evoked potential responses. The fact that the visual evoked potentials were bilaterally delayed suggests a general toxic/ adverse effect on the visual pathway. The pathogenesis of the damage is probably influenced by multiple factors: the tumor location, the dose and region of radiotherapy, the specific chemotherapeutic agents, increased intracranial pressure caused by hydrocephalus, and shunt dysfunctions and fluctuations of the intracranial pressure. The host factors (the patient's age at the time of diagnosis and treatment) may be critical as well.

An ophthalmological examination and a visual field testing of brain tumor survivors may be challenging due to confounding factors like difficulties with cooperation, cranial nerve palsies and problems with focusing and concentration. In this study, a third of children with abnormal visual evoked potentials had visual field defects. Visual field defects seemed to be more common in patients with abnormal visual evoked potentials, but 1 patient with scotomas and another patient with homonymous hemianopia had normal electroretinography and visual evoked potentials. Thus, normal study results do not exclude possible visual field defects. However, abnormal electroretinography and visual evoked potential tests indicate damage in the visual pathway and these tests may give information of a damaged visual pathway even when all other study results have been normal and before visual field defects or other clinical manifestations are visible. ${ }^{31}$ In 1 previous study with 9 nasopharyngeal carcinoma patients who had radiation-induced optic neuropathy, magnetic resonance imaging (MRI) showed enhancement of the optic nerve in 6 patients. ${ }^{32}$ Visual evoked potentials are more able to quantify the functional integrity of the optic pathways than scanning techniques such as MRI. ${ }^{15}$

Ocular coherence tomography is today routinely used in ophthalmology and is a good method for the evaluation of damage in anterior parts of the optic pathway. ${ }^{33}$ Ocular coherence tomography was not available during the study period. Visual evoked potential evaluates the entire optic pathway. ${ }^{31}$ Ocular coherence tomography and visual evoked potential could complement each other.

\section{Limitations}

Due to the heterogeneity of the study population, some subgroups evaluated were rather small. One limitation of the study was the lack of preoperative visual evoked potential and electroretinography in all patients. Only 3 patients underwent preoperative visual evoked potential and 1 patient had electroretinography. It is possible that some patients may have had preoperative abnormalities that may not be related to the brain tumor or tumor treatment. Six patients did not have ophthalmological testing, or the information was not available. In 4 cases, the visual fields could not be examined or the results were uncertain. Color vision testing of 9 patients was not available.

\section{Conclusions}

Electroretinographies were normal in all but 1 patient, but the incidence of visual evoked potential abnormalities was high even without evident anatomic lesions in the visual pathway. 
Many patients who did not have tumors involving the visual pathways had abnormal visual evoked potentials, including patients with infratentorial tumor location of whom more than one-third had abnormal visual evoked potentials. Abnormal visual evoked potentials indicate damage in the visual pathway and bilateral changes suggest a general and possibly multifactorial toxic/adverse effect on the visual pathway, which could also explain why abnormal visual evoked potentials were so common in patients with infratentorial tumor location. A longitudinal follow-up of these patients may be indicated as it is possible that abnormal visual evoked potential findings may be signs of later clinical features. The electroretinography and the visual evoked potential tests are functional and noninvasive studies that are easy and safe to repeat and may have both clinical and scientific value while evaluating long-term effects of childhood brain tumors and tumor treatment.

\section{Author Contribution}

SP, HLL, and AM designed the study. SP enrolled, screened, and examined the patients, collected and analyzed the data, and wrote the first draft. HLL and AM supervised the design. SO analyzed the electroretinography and visual evoked potential recordings. AMK supervised the statistical analysis of the data. TP functioned as a neurological consultant. HLL, SO, AMK, TP, and AM participated in the writing of the manuscript.

\section{Acknowledgments}

The authors thank warmly all the participants and their families, the personnel of the Department of Pediatrics and the Department of Child Neurology, Head-Nurse Mirja Uusitalo and the personnel of the Department of Clinical Neurophysiology, Docent Hannu Haapasalo, MD, of the Department of Pathology, Docent Pauli Helén, MD, of the Department of Neurosurgery, Raija Korpela, MD, PhD, of the Department of Child Neurology, Tuija Wigren, MD, PhD, of the Department of Oncology, and Juha Välimäki, MD, PhD, of the Department of Ophthalmology of Tampere University Hospital, and Jenny Junkkila, PsL, for language revision. This work was done at Tampere University Hospital and presented as a poster at the 46th Congress of the International Society of Paediatric Oncology (SIOP 2014) in Toronto, Canada, October 22-25, 2014.

\section{Declaration of Conflicting Interests}

The authors declared no potential conflicts of interest with respect to the research, authorship, and/or publication of this article.

\section{Funding}

The authors disclosed receipt of the following financial support for the research, authorship, and/or publication of this article: This work was supported by the Väre Foundation for Pediatric Cancer Research (grant decision 7.5.2013).

\section{Ethical Approval}

The study was approved by the Ethical Committee of Tampere University Hospital (approval number 98138) and was carried out with signed parental consent and/or patients' consent.

\section{References}

1. Steliarova-Foucher E, Stiller C, Kaatsch P, et al. Geographical patterns and time trends of cancer incidence and survival among children and adolescents in Europe since the 1970s (the ACCISproject): an epidemiological study. Lancet. 2004;364(9451):2097-2105.

2. Gatta G, Capocaccia R, Stiller C, et al. Childhood cancer survival trends in Europe: a EUROCARE Working Group study. J Clin Oncol. 2005;23(16):3742-3751.

3. Dickerman JD. The late effects of childhood cancer therapy. Pediatrics. 2007;119(5):554-568.

4. Pietilä S, Korpela R, Lenko HL, et al. Neurological outcome of childhood brain tumor survivors. J Neurooncol. 2012;108(1): 153-161.

5. Harbert MJ, Yeh-Nayre LA, O'Halloran HS, Levy ML, Crawford JR. Unrecognized visual field deficits in children with primary central nervous system brain tumors. J Neurooncol. 2012;107(3):545-549.

6. Chou SY, Digre KB. Neuro-ophthalmic complications of raised intracranial pressure, hydrocephalus, and shunt malfunction. $\mathrm{Neu}$ rosurg Clin N Am. 1999;10(4):587-608.

7. Nguyen TN, Polomeno RC, Farmer JP, Montes JL. Ophthalmic complications of slit-ventricle syndrome in children. Ophthalmology. 2002;109(3):520-525.

8. Mihalcea O, Arnold AC. Side effect of head and neck radiotherapy: optic neuropathy. Oftalmologia. 2008;52(1):36-40.

9. Behbehani R. Clinical approach to optic neuropathies. Clin Ophthalmol. 2007;1(3):233-246.

10. Lessell S. Friendly fire: neurogenic visual loss from radiation therapy. J Neuroophthalmol. 2004;24(3):243-250.

11. Gupta A, Dhawahir-Scala F, Smith A, Young L, Charles S. Radiation retinopathy: case report and review. BMC Ophthalmol. 2007;7:6.

12. Seregard S, Pelayes DE, Singh AD. Radiation therapy: posterior segment complications. Dev Ophthalmol. 2013;52:114-123.

13. You Y, Gupta VK, Li JC, Klistorner A, Graham SL. Optic neuropathies: characteristic features and mechanisms of retinal ganglion cell loss. Rev Neurosci. 2013;24(3):301-321.

14. Perlman I. The electroretinogram: ERG. In: Kolb H, Fernandez E, Nelson R, eds. Webvision: The Organization of the Retina and Visual System. Salt Lake City: University of Utah Health Sciences Center; 2007. Available at: http://webvision.med.utah.edu/book/ electrophysiology/the-electroretinogram-erg/. Accessed August $15,2014$.

15. Creel DJ. Visually evoked potentials. In: Kolb H, Fernandez E, Nelson R, eds. Webvision: The Organization of the Retina and Visual System. Salt Lake City: University of Utah Health Sciences Center; 2012. Available at: http://webvision.med.utah.edu/book/ electrophysiology/the-electroretinogram-erg/. Accessed August $15,2014$.

16. Falsini B, Ziccardi L, Lazzareschi I, et al. Longitudinal assessment of childhood optic gliomas: relationship between flicker visual evoked potentials and magnetic resonance imaging findings. J Neurooncol. 2008;88(1):87-96.

17. Kelly JP, Weiss AH. Detection of tumor progression in optic pathway glioma with and without neurofibromatosis type 1 . Neuro Oncol. 2013;15(11):1560-1567. 
18. Ilveskoski I, Saarinen UM, Perkkiö M, et al. Chemotherapy with the "8 in 1" protocol for malignant brain tumors in children: a population-based study in Finland. Pediatr Hematol Oncol. 1996; 13(1):69-80.

19. Zeltzer PM, Boyett JM, Finlay JL, et al. Metastasis stage, adjuvant treatment, and residual tumor are prognostic factors for medulloblastoma in children: conclusions from the Children's Cancer Group 921 randomized phase III study. J Clin Oncol. 1999;17(3):832-845.

20. Marmor MF. An updated standard for clinical electroretinography. Arch Ophthalmol. 1995;113(11):1375-1376.

21. Shurin SB, Rekate HL, Annable W. Optic atrophy induced by vincristine. Pediatrics. 1982;70(2):288-291.

22. O'Brien ME, Tonge K, Blake P, Moskovic E, Wiltshaw E. Blindness associated with high-dose carboplatin. Lancet. 1992; 339(8792):558.

23. Caraceni A, Martini C, Spatti G, Thomas A, Onofrj M. Recovering optic neuritis during systemic cisplatin and carboplatin chemotherapy. Acta Neurol Scand. 1997;96(4):260-261.

24. Wang MY, Arnold AC, Vinters HV, Glasgow BJ. Bilateral blindness and lumbosacral myelopathy associated with high-dose carmustine and cisplatin therapy. Am J Ophthalmol. 2000;130(3): 367-368.

25. Weisfeld-Adams JD, Dutton GN, Murphy DM. Vincristine sulfate as a possible cause of optic neuropathy. Pediatr Blood Cancer. 2007;48(2):238-240.
26. Wilding G, Caruso R, Lawrence TS, et al. Retinal toxicity after high-dose cisplatin therapy. J Clin Oncol. 1985;3(12):1683-1689.

27. Hilliard LM, Berkow RL, Watterson J, Ballard EA, Balzer GK, Moertel CL. Retinal toxicity associated with cisplatin and etoposide in pediatric patients. Med Pediatr Oncol. 1997;28(4): 310-313.

28. Krauss GL, Johnson MA, Miller NR. Vigabatrin-associated retinal cone system dysfunction: electroretinogram and ophthalmologic findings. Neurology. 1998;50(3):614-618.

29. Heim MK, Gidal BE. Vigabatrin-associated retinal damage: potential biochemical mechanisms. Acta Neurol Scand. 2012; 126(4):219-228.

30. York DH, Pulliam MW, Rosenfeld JG, Watts C. Relationship between visual evoked potentials and intracranial pressure. $J \mathrm{Neu}$ rosurg. 1981;55(6):909-916.

31. Laron M, Cheng H, Zhang B, Schiffman JS, Tang RA, Frishman LJ. Comparison of multifocal visual evoked potential, standard automated perimetry and optical coherence tomography in assessing visual pathway in multiple sclerosis patients. Mult Scler. 2010;16(4):412-426.

32. Zhao Z, Lan Y, Bai S, et al. Late-onset radiation-induced optic neuropathy after radiotherapy for nasopharyngeal carcinoma. $J$ Clin Neurosci. 2013;20(5):702-706.

33. Rodriguez-Mena D, Almarcegui C, Dolz I, et al. Electropysiologic evaluation of the visual pathway in patients with multiple sclerosis. J Clin Neurophysiol. 2013;30(4):376-381. 